\title{
Angiotensin-Converting Enzyme 2 Inhibits Apoptosis of Pulmonary Endothelial Cells During Acute Lung Injury Through Suppressing MiR-4262
}

\author{
Hong Bao Fengying Gao ${ }^{b}$ Guogang Xiec Zhenwei Liuc \\ aDepartment of Respiratory Medicine, Shanghai Pudong Hospital, Fudan University Pudong Medical \\ Center, Shanghai, 'bepartment of Respiratory Medicine, Shanghai JianGong Hospital, Shanghai, \\ 'Department of Respiratory Medicine, First People's Hospital Affiliated to Shanghai Jiaotong University, \\ Shanghai, China
}

\section{Key Words}

Acute lung injury (ALI) • Angiotensin converting enzyme 2 (ACE2) • Hydrolyzing angiotensin II (AngII) • Bcl-2 • MiR-4262

\begin{abstract}
Background/Aims: Angiotensin converting enzyme 2 (ACE2) treatment suppresses the severity of acute lung injury (ALI). The effects of ACE2 in ALI have been shown to not only result from its antagonizing hydrolyzing angiotensin II (AngII), which is responsible for reduction in the vascular tension and pulmonary accumulation of inflammatory cells, but also result from a role of ACE2 in suppressing the ALI-induced apoptosis of pulmonary endothelial cells (PECs). Nevertheless, the underlying mechanisms of the role of ACE2 on PEC apoptosis are not completely understood. Methods: Here, we used a bleomycin-induced mouse model for ALI that has been published in our previous studies. We analyzed the mRNA and protein levels of an anti-apoptotic protein Bcl-2 in the ALI-mice that have been treated w/o ACE2. We analyzed miR-4262 levels in the mouse lung in these mice. Bcl-2-targeting miRNAs were predicted using bioinformatics algorithms and a luciferase reporter assay was applied to examine the effects of miR-4262 on the Bcl-2 protein translation upon their binding to $3^{\prime}$ UTR of Bcl-2 mRNA. Adeno-associated viruses carrying either miR-4262 mimics or antisense were injected into ALI-mice without ACE2, and their effects on the apoptosis in mouse lung cells were analyzed by Western blot. Results: ACE2 inhibited the ALI-induced apoptosis of pulmonary cells in vivo partially through upregulation of Bcl-2 protein, but not Bcl-2 mRNA. ACE2 appeared to significantly suppress the upregulation of miR-4262 in mouse lung after ALI. MiR-4262 was found to target 3'-UTR of Bcl-2 mRNA to inhibit its protein translation in PECs. In vivo administration of antisense of miR-4262 decreased apoptosis of pulmonary cells and severity of the ALI in mice. Conclusion: ACE2-induced suppression of miR-4262 partially contribute to the inhibition of the PEC apoptosis after ALI through Bcl-2. MiR-4262 may be a novel promising treatment target for ALI and ARDS.




\section{Cellular Physiology Cell Physiol Biochem 2015;37:759-767 \\ and Bioch $\begin{array}{ll}\text { DOI: 10.1159/000430393 } 2015 \text { S. Karger AG, Basel }\end{array}$ \\ www.karger.com/cpb \\ Bao/Gao/Xie/Liu: ACE2 Inhibits ALI-Related PEC Apoptosis Through MiR-4262}

\section{Introduction}

Typical symptoms of acute lung injury (ALI) include expiratory dyspnea, refractory hypoxemia and non-cardiogenic pulmonary edema, the deterioration of which could result in the aggravation of ALI into highly lethal Acute Respiratory Distress Syndrome (ARDS) [1-4]. The cellular pathology of ALI is comprised of loss of alveolar-capillary membrane integrity, excessive transepithelial neutrophil migration and release of pro-inflammatory cytokines, e.g. interleukin (IL)-6 and tumor necrosis factor (TNF)- $\alpha$ [1-4]. Injuries of both pulmonary endothelial cells (PECs) and alveolar epithelial cells occur after ALI, resulting in loss of respiratory capacity [1-4].

Being primary perpetrators of inflammation, neutrophils and their migration cross the vascular epithelia are critical for development of ALI [1-4]. Excessive and prolonged activation of neutrophils results in basement membrane destruction and increased permeability of the alveolar-capillary barrier [1-4]. Moreover, neutrophils also release pro-inflammatory and pro-apoptotic cytokines to injure adjacent cells to create ulcerating lesions to aggravate the damages of the alveolar-capillary barrier [1-4].

Angiotensin-converting enzyme 2 (ACE2) belongs to the renin-angiotensin system [5], in which renin induces the production of angiotensin I (Ang I), which is converted to an important vasoconstrictive peptide Ang II by Ang I-converting enzyme (ACE) [5]. ACE2 is the homolog of ACE but counterbalances the ACE activity through induction of degradation of Ang II [5]. Recently, we and others have shown that ACE2 has therapeutic effects on ALI, at least partially through suppression of apoptosis of PECs [6-10]. However, the molecular mechanisms underlying the inhibitory effects of ACE2 on cell apoptosis are not completely understood.

Bcl-2 (B-cell lymphoma 2) is an important anti-apoptotic protein and it binds to the apoptotic proteins BAD and BAK to inhibit their functions. Apoptosis plays a very active role in regulating the immune system [11-13]. When $\mathrm{Bcl}-2$ is functional, it can cause immune unresponsiveness to self-antigens via both central and peripheral tolerance [11-13]. In the case of defective apoptosis, it may contribute to etiological aspects of autoimmune diseases [11-13]. We have previously found that ACE2 induced upregulation of Bcl-2 protein in the mouse lung after ALI [9], whereas the mechanisms remain to be determined.

Here, we showed that ACE2 inhibited the ALI-induced apoptosis of pulmonary cells in vivo partially through upregulation of Bcl-2 protein, but not Bcl-2 mRNA. ACE2 appeared to significantly suppress the upregulation of miR-4262 in mouse lung after ALI. MiR-4262 was found to target 3'-UTR of Bcl-2 mRNA to inhibit its protein translation in PECs. In vivo administration of antisense of miR-4262 decreased apoptosis of pulmonary cells and severity of the ALI in mice. These data suggest that ACE2-induced suppression of miR-4262 may partially contribute to the inhibition of the PEC apoptosis after ALI through Bcl-2.

\section{Materials and Methods}

\section{Animal manipulations}

All mouse experiment protocols were approved by the Animal Research and Care Committee at First people's Hospital Affiliated to Shanghai Jiaotong University. All experiments were performed in accordance with the guidelines from the Animal Research and Care Committee at First people's Hospital Affiliated to Shanghai Jiaotong University.

Specific pathogen free (SPF) Balb/c mice (aged 10 weeks, weight 18-22g) were supplied by Laboratory Animal Center of Shanghai Academy of Sciences, Chinese Academy of Sciences, China. After acclimatization for 1 week, a group of mice were used as the no-injury group and received no treatments (NT). The lung injury model was induced in another groups of mice, by intraperitoneal administration of the bleomycin solution (10mg/mL, Sigma-Aldrich, St Louis, MO, USA) at $20 \mathrm{mg} / \mathrm{kg}$ body weight, as has been applied and described before $[6-10,14]$. This ALI model is stable and easy to be built. No death was observed in the current study. After successful induction of ALI, the mice were further randomly divided into groups to 


\section{Cellular Physiology Cell Physiol Biochem 2015;37:759-767

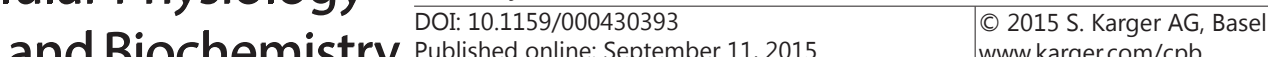 www.karger.com/cpb \\ Bao/Gao/Xie/Liu: ACE2 Inhibits ALI-Related PEC Apoptosis Through MiR-4262}

receive single tail vein injection of either saline (termed ALI), or ACE2 (R\&D systems, Shanghai, China) at a dose of $1 \mathrm{mg} / \mathrm{kg}$ body weight (termed ALI+ACE2), or $10^{9}$ adeno-associated virus (AAV) carrying null construct (ALI+AAV-null), or $10^{9} \mathrm{AAV}$ carrying antisense of miR-4262 (ALI+AAV-as-miR-4262). Ten mice were used in one experimental group.

\section{Cell lines}

Human embryonic kidney 293 cell line (HEK293) and Human lung epithelial cell line HULEC-5a (HULEC) were purchased from ATCC (American Type Culture Collection, Manassas, VA, USA), and has been described before [9]. HULEC-5a is an immortalized human microvascular endothelial cell Line [15], and were maintained in MCDB131 media (without L-Glutamine, ATCC) supplemented with 10ng/ml Epidermal Growth Factor (EGF, Sigma-Aldrich), $1 \mu \mathrm{g} / \mathrm{ml}$ Hydrocortisone (Sigma-Aldrich), 10mmol/l Glutamine (Invitrogen, Carlsbad, CA, USA) and 10\% fetal bovine serum (FBS, Invitrogen). Both cells were incubated in a humidified chamber with $5 \% \mathrm{CO}_{2}$ at $37^{\circ} \mathrm{C}$.

\section{AAV preparation and cell transfections}

AAV-as-miR-4262 and control AAV-null viruses were prepared according to general protocols. Briefly, we used a pAAV-CMV-GFP plasmid (Clontech, Mountain View, CA, USA), a packaging plasmid carrying the serotype 8 rep and cap genes, and a helper plasmid carrying the adenovirus helper functions (Applied Viromics, LLC. Fremont, CA, USA) in this study. The transgenes as-miR-4262 or null were inserted between CMV promoter and GFP reporter. 2A sequence was used to connect transgene and GFP to allow co-expression of both under the control of the CMV promoter. The transgenes were subcloned into the plasmid using the 50-EcoRI and 30-NheI sites of the pAAV-CMV-GFP vector. Sequencing was performed to confirm the correct orientation of the generated plasmids. AAVs were prepared by triple transfection of the newly prepared plasmids, R2C8 (containing AAV2 Rep and AAV8 capsid genes) and plAd5 (containing adenovirus helper genes) into HEK293 cells by Lipofectamine 2000 reagent (Invitrogen). The viruses were purified using $\mathrm{CsCl}$ density centrifugation and then titered by a quantitative densitometric dot-blot assay. For cell transfection in vitro, the HULEC cells were incubated with plasmids (miR-4262, as-miR-4262 and null) at a MOI of 100 for 12 hours.

RNA extraction, reverse transcription and quantitative RT-PCR (RT-qPCR)

Total RNA and miRNAs were extracted from lung or from the cultured cells with miRNeasy mini kit or RNeasy kit (Qiagen, Hilden, Germany), respectively. Complementary DNA (cDNA) was randomly primed from $2 \mu \mathrm{g}$ of total RNA using the Omniscript reverse transcription kit (Qiagen). RT-qPCR was subsequently performed in triplicate with a 1:4 dilution of cDNA using the Quantitect SyBr green PCR system (Qiagen) on a Rotorgene 6000 series PCR machine. Quantitative PCR (RT-qPCR) were performed in duplicates with QuantiTect SYBR Green PCR Kit (Qiagen). All primers were purchased from Qiagen. Data were collected and analyzed with the Rotorgene software accompanying the PCR machine, using 2- $\Delta \mathrm{Ct}$ method for quantification of the relative mRNA expression levels. Values of genes were first normalized against $\alpha$-tubulin, and then compared to controls.

\section{Western blot}

The protein from the mouse lung or cultured cells was extracted using RIPA lysis buffer (1\% NP40, $0.1 \%$ Sodium dodecyl sulfate (SDS), $100 \mu \mathrm{g} / \mathrm{ml}$ phenylmethylsulfonyl fluoride, $0.5 \%$ sodium deoxycholate, in PBS) on ice. The supernatants were collected after centrifugation at $12000 \times \mathrm{g}$ at $4^{\circ} \mathrm{C}$ for $20 \mathrm{~min}$. Protein concentration was determined using a BCA protein assay kit (Bio-rad, China), and whole lysates were mixed with $4 \times$ SDS loading buffer (125mmol/l Tris-HCl, 4\% SDS, $20 \%$ glycerol, $100 \mathrm{mmol} / \mathrm{l}$ Dithiothreitol (DTT), and $0.2 \%$ bromophenol blue) at a ratio of $1: 3$. Samples were heated at $100{ }^{\circ} \mathrm{C}$ for 5 min and were separated on SDS-polyacrylamide gels. The separated proteins were then transferred to a PVDF membrane. The membrane blots were first probed with a primary antibody. After incubation with horseradish peroxidaseconjugated second antibody, autoradiograms were prepared using the enhanced chemiluminescent system to visualize the protein antigen. The signals were recorded using X-ray film. Primary antibodies were antitumor necrosis factor $\alpha$ (TNF $\alpha$ ), anti-IL6, anti-CXCL1, anti-Cytochrome C (CytoC), anti-caspase9 (casp9), anti-Bcl-2 and $\alpha$-tubulin (all purchased from Cell Signaling, St Louis, MO, USA). $\alpha$-tubulin was used as a protein loading control. Secondary antibody is HRP-conjugated anti-rabbit (Jackson ImmunoResearch Labs, 


\section{Cellular Physiology Cell Physiol Biochem 2015;37:759-767

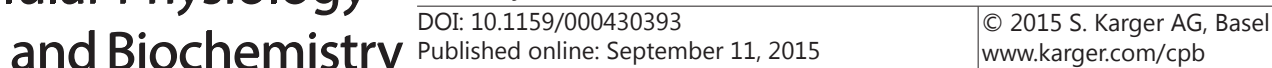 \\ Bao/Gao/Xie/Liu: ACE2 Inhibits ALI-Related PEC Apoptosis Through MiR-4262}

West Grove, PA, USA). Images shown in the figure were representative from 5 repeats. Densitometry of Western blots was quantified with NIH ImageJ software (Bethesda, MA, USA). The protein levels were first normalized to loading controls, and then normalized to experimental controls.

Bioinformatic analyses and luciferase-reporter activity assay

Targeting miRNAs for 3'-UTR of Bcl-2 mRNA were predicted using the following algorithms: TargetScan (https://www.targetscan.org) [16]. Luciferase-reporters were successfully constructed using molecular cloning technology. Target sequence for Bcl-2 miRNA 3'UTR clone was purchased from Creative Biogene (Shirley, NY, USA). HULEC-miR-4262, or HULEC-null, or HULEC-as-miR-4262 cells were seeded in 24-well plates for 24 hours, after which they were transfected with $1 \mu \mathrm{g}$ of Luciferase-reporter plasmids per well using PEI Transfection Reagent. Luciferase activities were measured using the dual-luciferase reporter gene assay kit (Promega, Beijing, China), according to the manufacturer's instructions.

Statistical analysis

All statistical analyses were carried out using the SPSS 18.0 statistical software package. All values are depicted as mean \pm standard deviation from 5 individuals and are considered significant if $\mathrm{p}<0.05$. All data were statistically analyzed using one-way ANOVA with a Bonferoni correction, followed by a Fisher's exact test, as necessary.

\section{Results}

ACE2 reduces ALI features in mouse lung

The mouse bleomycin-ALI model has been performed as has been described before [6$10,14]$. H\&E staining of mouse lung tissue showed significant pneumonitis, inflammatory exudates, fibroblastic foci and distortion of the normal architecture of the lung after ATI, while ACE2 reduced the severity of these features (Fig. 1A). As a routine quality control, we isolated protein from the lung of the mice treated with bleomycin and ACE2 (ALI+ACE2) and then analyzed the inflammatory cytokine levels by Western blot, compared to the mice without treatment (UT) and to the mice treated with bleomycin and control saline (ALI). We found that ALI induced significantly increases in TNF $\alpha$, IL6 and CXCL1 levels, by quantification (Fig. 1B), and by representative images (Fig. 1C). These data confirm the successful establishment of mouse ALI model and the effects of ACE2 against ALI severity.

ACE2 increases Bcl-2 protein but not mRNA in mouse lung after ALI

We have previously shown that ACE2 reduces cell apoptosis in mouse lung after ALI. Now we studied the underlying mechanisms. Among all examined pro-apoptotic proteins and anti-apoptotic proteins, we specifically found that although the mRNA levels of Bcl2 remained unchanged by ACE2 (Fig. 2A), the protein levels of Bcl-2 were significantly increased by ACE2 administration (Fig. 2B). These data suggest that ACE2 may induce the translational control of Bcl-2 in mouse lung after ALI.

\section{ACE2 reduces miR-4262, which suppresses Bcl-2 translation}

Since miRNAs often regulate translation of mRNA through binding to the 3'-UTR of mRNA of certain genes, we screened all Bcl-2 targeting miRNAs, and we found that miR4262 levels in the mouse lung were significantly decreased by ACE2 (Fig. 3A).

We then performed bioinformatics analysis of 3'-UTR of Bcl-2 mRNA target sequence, which showed that the miR-4262 binding sites in the 3'-UTR of Bcl-2 mRNA ranged from 2896th base site to 2903th base site (Fig. 3B). In order to prove that this binding is functional, we modified miR-4262 levels in HULEC cells, and these HULEC-miR-4262, HULEC-null (as a control) and HULEC-as-miR-4262 cells were confirmed of changes in miR-4262 levels (Fig. 3C). These cells were then transfected with $1 \mu \mathrm{g}$ of Bcl-2-3'UTR luciferase-reporter plasmid. The luciferase activities in these cells suggest that miR-4262 targets 3'UTR of Bcl-2 mRNA to inhibit its expression (Fig. 3D).

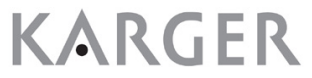




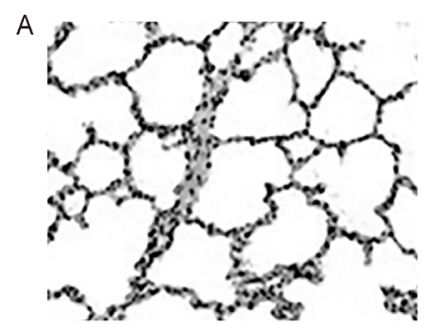

UT

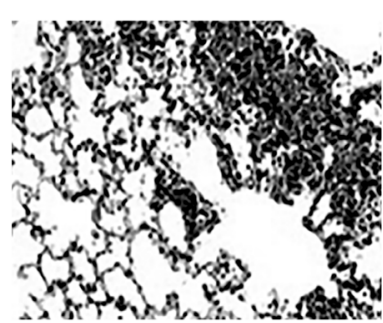

ALI

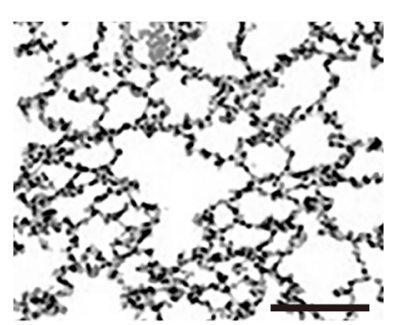

ALI+ACE2
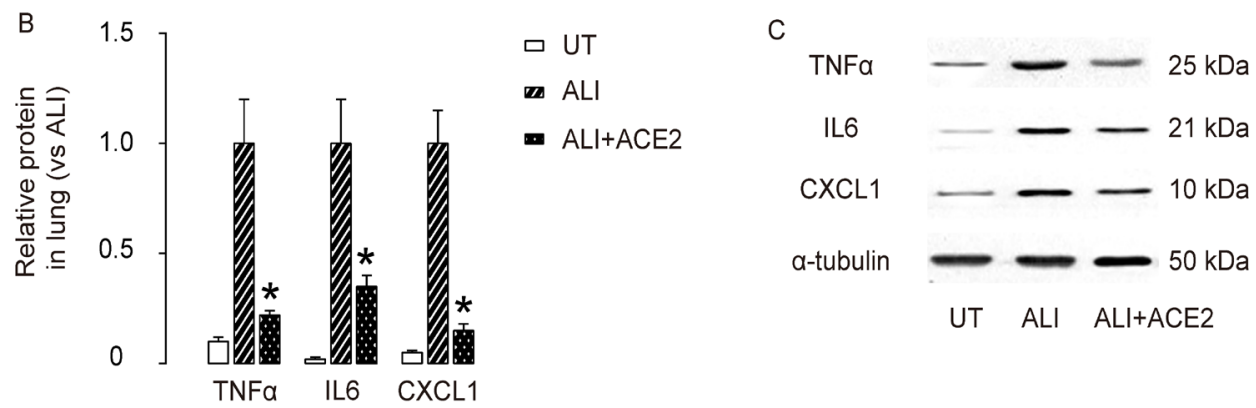

Fig. 1. ACE2 significantly reduces the severity of lung injury by bleomycin. (A) Representative HE staining of lung tissue. (B-C) We isolated protein from the lung of the mice treated with bleomycin and ACE2 (ALI+ACE2) and then analyzed the inflammatory cytokine levels by Western blot, compared to the mice without treatment (UT) and to the mice treated with bleomycin and saline (ALI). The data were shown by quantification (B), and by representative images (C). ${ }^{*} \mathrm{p}<0.05$ (ALI+ACE2 vs ALI). N=10. Statistics: one-way ANOVA, followed by Fisher's exact test. Scale bar is $50 \mu \mathrm{m}$.
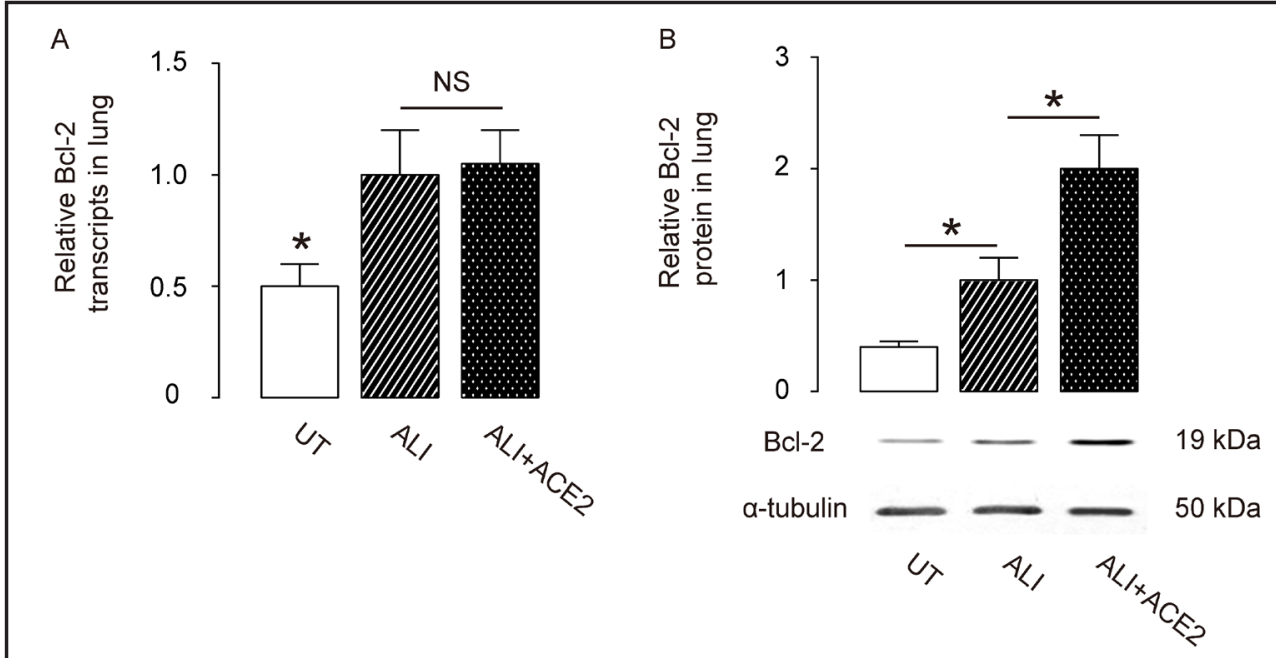

Fig. 2. ACE2 increases Bcl-2 protein but not mRNA in mouse lung after ALI. (A-B) Among all examined pro-apoptotic proteins and anti-apoptotic proteins, we specifically found that although the mRNA levels of Bcl-2 remained unchanged by ACE2 (A), the protein levels of Bcl-2 were significantly increased by ACE2 administration (B). ${ }^{*} \mathrm{p}<0.05$. NS: non-significant. $\mathrm{N}=10$. Statistics: one-way ANOVA, followed by Fisher's exact test.

In vivo administration of as-miR-4262 mimics the effects of ACE2 on cell apoptosis and ALI features

In order to confirm that the effects of ACE2 on apoptosis-related proteins in ALI lung is partially conducted through miR-4262-regualted $\mathrm{Bcl}-2$ translation, we prepared AAVs 


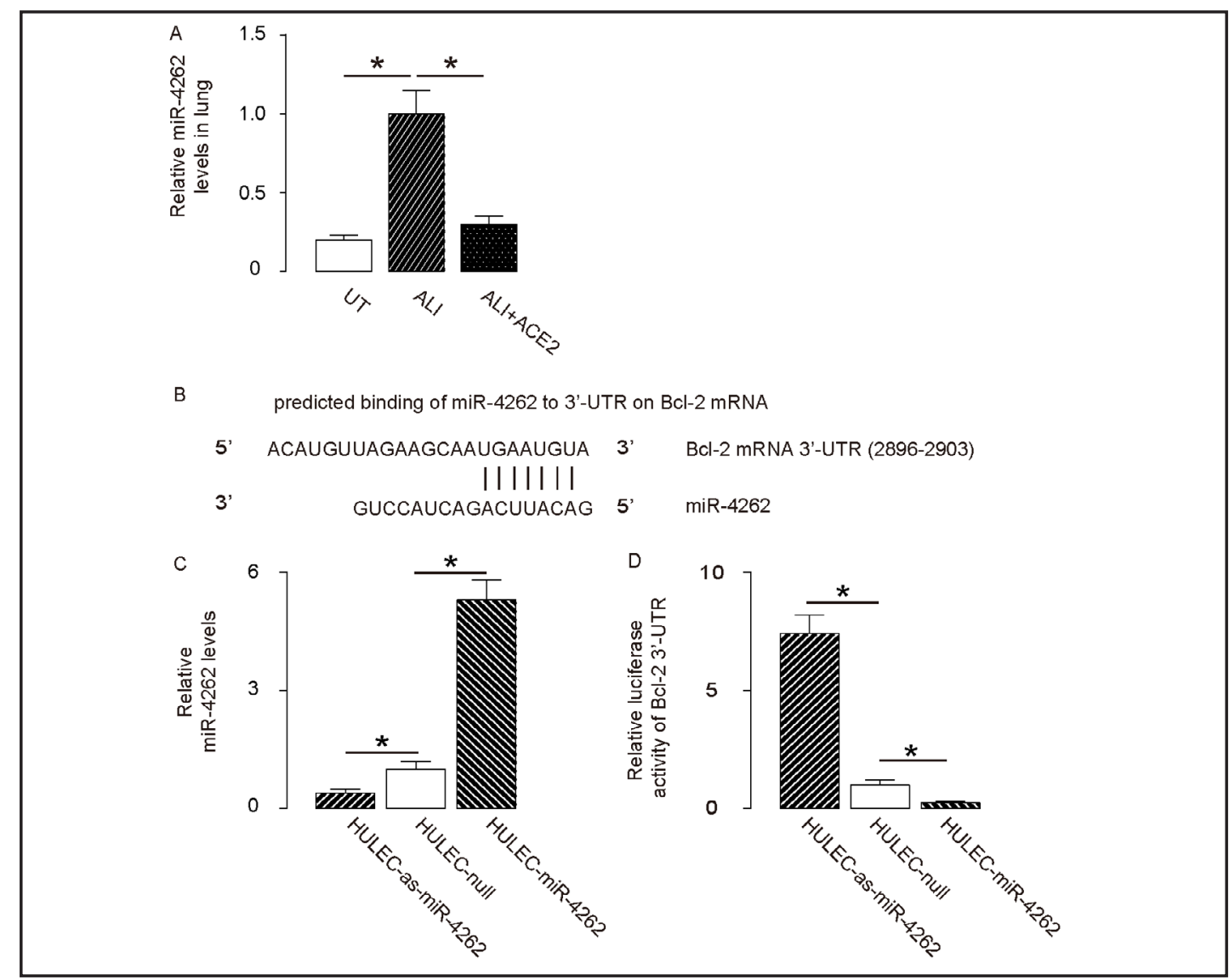

Fig. 3. ACE2 reduces miR-4262, which suppresses Bcl-2 translation. (A) MiR-4262 levels in the mouse lung were significantly decreased by ACE2. (B)Bioinformatics analysis of 3'-UTR of Bcl-2 mRNA target sequence, showing that the miR-4262 binding sites in the 3'-UTR of Bcl-2 mRNA ranged from 2896th base site to 2903th base site. (C) The modification of miR-4262 levels in HULEC cells (HULEC-miR-4262, HULEC-null and HULEC-as-miR-4262 cells) were confirmed by RT-qPCR. (D) The miR-4262-modified cells were then transfected with $1 \mu \mathrm{g}$ of Bcl-2-3'UTR luciferase-reporter plasmid and analyzed for the luciferase activities. ${ }^{*} \mathrm{p}<0.05$. $\mathrm{N}=10$. Statistics: one-way ANOVA, followed by Fisher's exact test.

carrying as-miR-4262 and control AAVs carrying null. These AAVs were injected instead of ACE2 to the mice.

H\&E staining of mouse lung tissue showed significant improvement of the severity of ALI by AAV-as-miR-4262 (Fig. 4A). The administration of AAV-as-miR-4262 significantly reduced miR-4262 levels in the mouse lung after ALI (Fig. 4B). Moreover, although administration of AAV-as-miR-4262 did not change the Bcl-2 transcript levels (Fig. 4B), it significantly increased the Bcl-2 proteins, and significantly reduced the levels of the inflammatory cytokines TNF $\alpha$, IL6 and CXCL1, as well as significantly reduced apoptotic proteins Cytochrome C (CytoC) and caspase 9 (casp9) (Fig. 4C). Thus, our data suggest that ACE2-induced suppression of miR4262 may partially contribute to the inhibition of the PEC apoptosis after ALI through Bcl-2. Thus, this model was summarized in a schematic (Fig. 5).

\section{Discussion}

ALI-induced ARDS is one of the leading causes for the death of patients in intense care unit. The current ALI therapy mainly applies supportive treatments, such as protective ventilation. However, these supportive treatments are only effective to less severe subjects, 


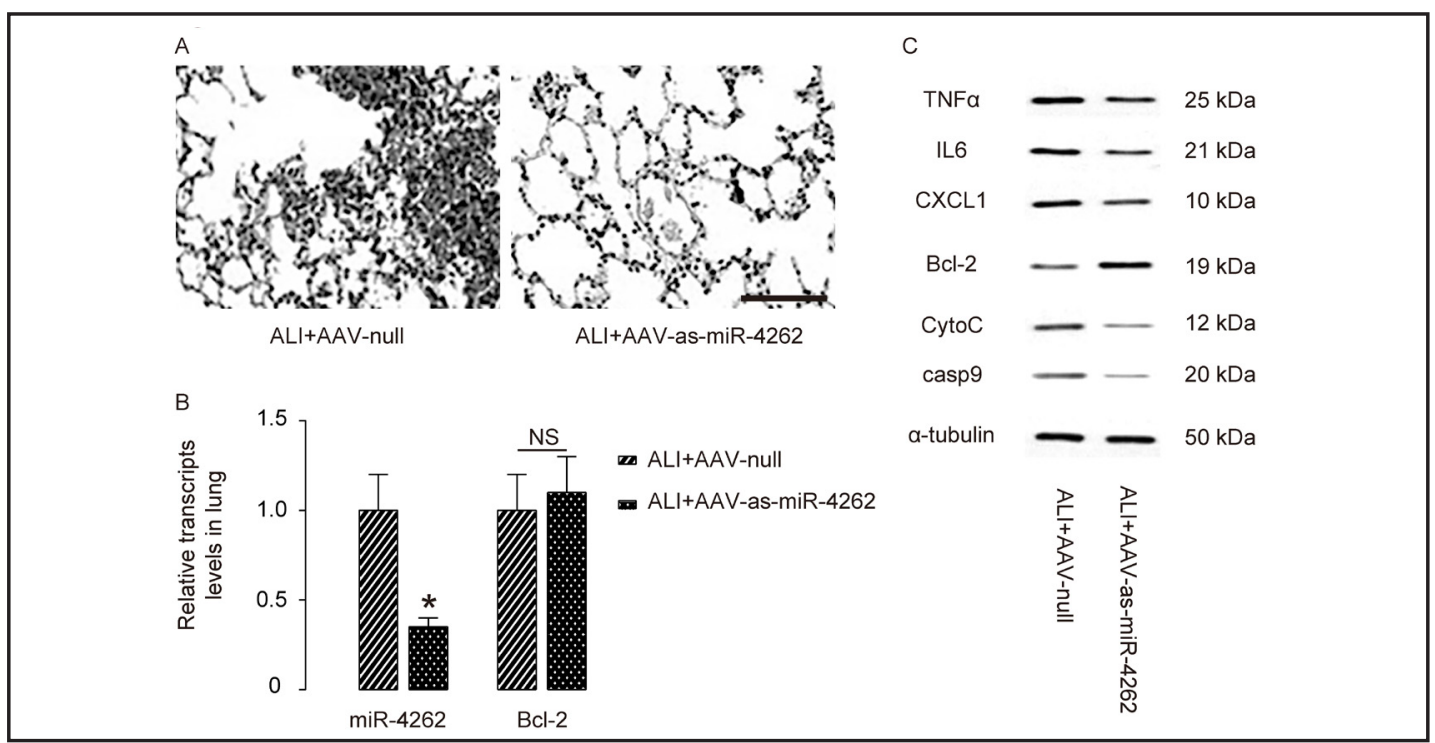

Fig. 4. In vivo administration of as-miR-4262 mimics the effects of ACE2 on cell apoptosis and ALI features. In order to confirm that the effects of ACE2 on apoptosis-related proteins in ALI lung is partially conducted through miR-4262-regualted Bcl-2 translation, we prepared AAVs carrying as-miR-4262 and control AAVs carrying null. These AAVs were injected instead of ACE2 to the mice. (A) H\&E staining of mouse lung tissue showed significant improvement of the severity of ALI by AAV-as-miR-4262. (B) The transcript levels for miR-4262 and Bcl-2. (C) Representative Western blot images for Bcl-2, TNF $\alpha$, IL6, CXCL1, Cytochrome C (CytoC) and caspase 9 (casp9). ${ }^{*} \mathrm{p}<0.05$. NS: non-significant. $\mathrm{N}=10$. Statistics: one-way ANOVA, followed by Fisher's exact test.

Fig. 5. Schematic of the model. ACE2induced suppression of miR-4262 may partially contribute to the inhibition of the PEC apoptosis after ALI through Bcl-2.

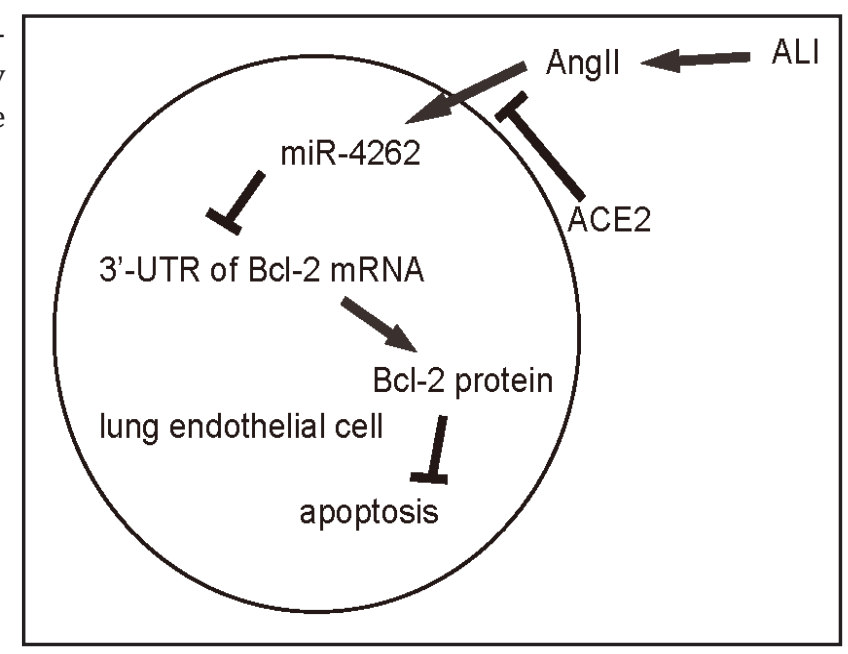

and their effects on severe cases are quite limited [1-4]. Hence, innovative therapy is highly needed to improve the current ALI/ARDS treatments, and this therapeutic innovation requires better understanding the molecular pathology of ALI and requires identification of new targets.

To date, it is well-known that ALI is characterized with loss of alveolar-capillary membrane integrity, excessive transepithelial neutrophil migration and release of proinflammatory cytokines [1-4]. ALI damages on both PECs and alveolar epithelial cells result in loss of respiratory capacity [1-4]. Moreover, the damages to PECs appear to occur ahead of the damages to epithelial cells [17].

The protective roles of ACE2 in acute and chronic lung diseases have been demonstrated, including anti-inflammation and anti-oxidation. In addition, we recently reported a role of 


\section{Cellular Physiology Cell Physiol Biochem 2015;37:759-767

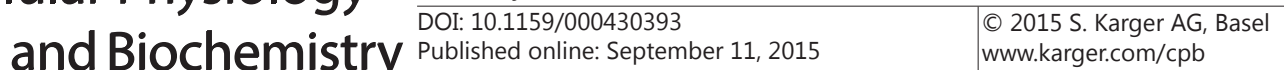 \\ Bao/Gao/Xie/Liu: ACE2 Inhibits ALI-Related PEC Apoptosis Through MiR-4262}

ACE2 in inhibiting cell apoptosis after ALI [9]. However, the mechanisms underlying this regulation remain undetermined.

Here we confirmed that ACE2 significantly increased the protein levels of anti-apoptotic protein Bcl-2 in mouse lung after ALI. Moreover, we found that ACE2 did not alter Bcl-2 mRNA levels. These findings suggest presence of a regulation of Bcl-2 at post-transcriptional level. Then we screened all Bcl-2 targeting miRNAs, including miR-21, miR-204, miR-211, miR-590, miR-449, miR-34, miR-125, miR-195 and miR-4262 etc, we specifically found that miR-4262 levels were significantly altered by ACE2 treatment. The regulation of Bcl-2 translation by miR-4262 was confirmed using luciferase reporter assay.

$\mathrm{Bcl}-2$ proteins have an $\mathrm{N}$-terminal amino acid residue (K17) required for their interaction with IP3R to coordinate Calcium uptake into mitochondria to affect apoptosis [18-20]. There is recent evidence that the $\mathrm{Na} / \mathrm{K}$ pump interacts with Bcl-2 proteins and thus with IP3R [21]. The involvement of this mechanisms of the current model should be tested in future.

Since the direct target of ACE2 in lung is Ang II, the receptor of which is uniquely expressed in PECs, we have shown that the direct effects of ACE2 should be Ang II-related, and most likely to be conducted on PECs [9]. Thus, we used HULEC cells in the luciferase reporter assay to confirm the functional binding of miR-4262 to the 3'-UTR of Bcl-2 mRNA.

In our previous study, we found that ALI induced phosphorylation of a key factor SMAD2 of TGF $\beta$ receptor signaling [22-28], which could be dose-dependently inhibited by ACE2. Hence, it is possible that the regulation of miR-4262 by ACE2 could be through modulation of TGF $\beta$ receptor signaling. Indeed, we examined the miR-4262 levels in the mouse lung in SMAD7-overexpressing model and found that SMAD7 overexpression inhibited increases in miR-4262 levels by ALI. On the other hand, it is possible that ACE2 may induce production of non-functional ATII breakdown products, i.e. ATIII-V, to exert its function. That way, an ATII receptor blocker should have similar effects as ACE2? This hypothesis may be examined in the future experiment.

Further experiments may be also applied to examine the protective roles of ACE2 besides its inhibition on apoptosis, and to determine the targets of miR-4262 other than Bcl-2. These approaches may greatly improve our understandings of the molecular basis of ACE2-mediated protection against ALI, and may shed light on development of novel therapies.

\section{Disclosure Statement}

The authors have declared that no conflict of interest exists.

\section{References}

1 Ashbaugh DG, Bigelow DB, Petty TL, Levine BE: Acute respiratory distress in adults. Lancet 1967;2:319323.

2 Herold S, Gabrielli NM, Vadasz I: Novel concepts of acute lung injury and alveolar-capillary barrier dysfunction. Am J Physiol Lung Cell Mol Physiol 2013;305:L665-681.

3 Dengler V, Downey GP, Tuder RM, Eltzschig HK, Schmidt EP: Neutrophil intercellular communication in acute lung injury. Emerging roles of microparticles and gap junctions. Am J Respir Cell Mol Biol 2013;49:15.

4 Vlaar AP, Juffermans NP: Transfusion-related acute lung injury: A clinical review. Lancet 2013;382:984994.

5 Imai Y, Kuba K, Penninger JM: The discovery of angiotensin-converting enzyme 2 and its role in acute lung injury in mice. Exp Physiol 2008;93:543-548.

6 Chen D, Jiao G, Ma T, Liu X, Yang C, Liu Z: The mechanism of rapamycin in the intervention of paraquatinduced acute lung injury in rats. Xenobiotica 2014:1-9.

7 Liu F, Gao F, Li Q Liu Z: The functional study of human umbilical cord mesenchymal stem cells harbouring 


\section{Cellular Physiology Cell Physiol Biochem 2015;37:759-767 \begin{tabular}{l|l|l}
\hline DOI: 10.1159/000430393 & (C) 2015 S. Karger AG, Basel
\end{tabular} \\ Bao/Gao/Xie/Liu: ACE2 Inhibits ALI-Related PEC Apoptosis Through MiR-4262}

angiotensin-converting enzyme 2 in rat acute lung ischemia-reperfusion injury model. Cell Biochem Funct 2014;32:580-589.

8 Liu D, Dong Y, Liu Z, Niu B, Wang Y, Gao X: Impact of trem-2 gene silencing on inflammatory response of endotoxin-induced acute lung injury in mice. Mol Cell Biochem 2014;394:155-161.

9 Ji Y, Gao F, Sun B, Hao J, Liu Z: Angiotensin-converting enzyme 2 inhibits apoptosis of pulmonary endothelial cells during acute lung injury through suppressing smad2 phosphorylation. Cell Physiol Biochem 2015;35:2203-2212.

10 Min F, Gao F, Li Q, Liu Z: Therapeutic effect of human umbilical cord mesenchymal stem cells modified by angiotensin-converting enzyme 2 gene on bleomycin-induced lung fibrosis injury. Mol Med Rep 2015;11:2387-2396.

11 Kim Y, Wang M, Bai Y, Zeng Z, Guo F, Han N, Bian H, Wang J, Pan J, Zhu M: Bcl-2 suppresses activation of vpes by inhibiting cytosolic ca(2)(+) level with elevated $\mathrm{k}(+)$ efflux in nacl-induced pcd in rice. Plant Physiol Biochem 2014;80:168-175.

12 Zhu Y, Wu J, Li S, Ma R, Cao H, Ji M, Jing C, Tang J: The function role of mir-181a in chemosensitivity to adriamycin by targeting bcl-2 in low-invasive breast cancer cells. Cell Physiol Biochem 2013;32:1225-1237.

13 Lauf PK, Heiny J, Meller J, Lepera MA, Koikov L, Alter GM, Brown TL, Adragna NC: Canonical bcl-2 motifs of the na+/k+ pump revealed by the bh3 mimetic chelerythrine: Early signal transducers of apoptosis? Cell Physiol Biochem 2013;31:257-276.

14 Liu W, Shan LP, Dong XS, Liu Z: Toll-like receptor 4 implicated in acute lung injury induced by paraquat poisoning in mice. International journal of clinical and experimental medicine 2014;7:3392-3397.

15 Ades EW, Candal FJ, Swerlick RA, George VG, Summers S, Bosse DC, Lawley TJ: Hmec-1: Establishment of an immortalized human microvascular endothelial cell line. J Invest Dermatol 1992;99:683-690.

16 Coronnello C, Benos PV: Comir: Combinatorial microrna target prediction tool. Nucleic Acids Res 2013;41:W159-164.

17 Bader AM, Brodarac A, Klose K, Bieback K, Choi YH, Kang KS, Kurtz A, Stamm C: Cord blood mesenchymal stromal cell-conditioned medium protects endothelial cells via stat3 signaling. Cell Physiol Biochem 2014;34:646-657.

18 Vervliet T, Decrock E, Molgo J, Sorrentino V, Missiaen L, Leybaert L, De Smedt H, Kasri NN, Parys JB, Bultynck G: Bcl-2 binds to and inhibits ryanodine receptors. J Cell Sci 2014;127:2782-2792.

19 Akl H, Bultynck G: Altered ca(2+) signaling in cancer cells: Proto-oncogenes and tumor suppressors targeting ip3 receptors. Biochim Biophys Acta 2013;1835:180-193.

20 Decuypere JP, Monaco G, Missiaen L, De Smedt H, Parys JB, Bultynck G: Ip(3) receptors, mitochondria, and ca signaling: Implications for aging. J Aging Res 2011;2011:920178.

21 Monaco G, Decrock E, Arbel N, van Vliet AR, La Rovere RM, De Smedt H, Parys JB, Agostinis P, Leybaert L, Shoshan-Barmatz V, Bultynck G: The bh4 domain of anti-apoptotic bcl-xl, but not that of the related bcl-2, limits the voltage-dependent anion channel 1 (vdac1)-mediated transfer of pro-apoptotic ca2+ signals to mitochondria. J Biol Chem 2015;290:9150-9161.

22 Stopa M, Anhuf D, Terstegen L, Gatsios P, Gressner AM, Dooley S: Participation of smad2, smad3, and smad4 in transforming growth factor beta (tgf-beta)-induced activation of smad7. The tgf-beta response element of the promoter requires functional smad binding element and e-box sequences for transcriptional regulation. J Biol Chem 2000;275:29308-29317.

23 Ulloa L, Doody J, Massague J: Inhibition of transforming growth factor-beta/smad signalling by the interferon-gamma/stat pathway. Nature 1999;397:710-713.

24 Xiao X, Gaffar I, Guo P, Wiersch J, Fischbach S, Peirish L, Song Z, El-Gohary Y, Prasadan K, Shiota C, Gittes GK: M2 macrophages promote beta-cell proliferation by up-regulation of smad7. Proc Natl Acad Sci U S A 2014;111:E1211-1220.

25 Yu Y, Ran Q: Nuclear smad2 restrains proliferation of glioblastoma. Cell Physiol Biochem 2015;35:17561763.

26 Huang S, Liao Q, Li L, Xin D: Pttg1 inhibits smad3 in prostate cancer cells to promote their proliferation. Tumour Biol 2014;35:6265-6270.

27 Sun R, Luo Y, Li J, Wang Q Li J, Chen X, Guan K, Yu Z: Ammonium chloride inhibits autophagy of hepatocellular carcinoma cells through smad2 signaling. Tumour Biol 2015;36:1173-1177.

28 Xia Q, Li C, Bian P, Wang J, Dong S: Targeting smad3 for inhibiting prostate cancer metastasis. Tumour Biol 2014;35:8537-8541. 